\title{
Comparison of RIP, EIGRP, OSPF, IGRP Routing Protocols in Wireless Local Area Network (WLAN) by using OPNET Simulator tool - A Practical Approach
}

\author{
P. Kalamani ${ }^{1}$, M. Venkatesh Kumar ${ }^{2}$, M. Chithambarathanu ${ }^{3}$, Reji Thomas ${ }^{4}$

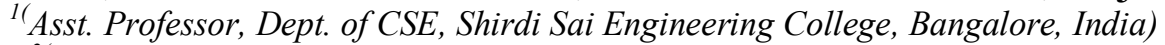 \\ ${ }^{2}$ Sr. Lecturer, Dept. of CSE, Shirdi Sai Engineering College, Bangalore, India) \\ ${ }^{3}$ (Asst. Professor, Dept. of CSE, Shirdi Sai Engineering College, Bangalore, India) \\ ${ }^{4}$ Sr. Lecturer, Dept. of CSE, Shirdi Sai Engineering College, Bangalore, India)
}

\begin{abstract}
In Adhoc Network group communication is more important, in which routing protocols play a vital role for data transmission. With/Without using central server or access point, the Wireless network form a temporary network with collection of wireless nodes in which, each node changes randomly at different times. In order to establish data transmission between nodes, multiple hops are needed because of limited range i.e. transmission rate. In this paper, we have analyzed and simulated a proposed Wireless Local Area Network (WLAN) using different routing protocols. The performances of different protocols are compared and analyzed using Optimum Network Performance (OPNET) simulator tool in which metrics like delay, throughput, packet delivery, load, Ethernet delay, are measured.
\end{abstract}

Keywords: Adhoc Networks, OPNET, WLAN, RIP, OSPF, IGRP, EIGRP, Routing Protocol

\section{Introduction}

Nodes communicate with one another by using multi-hop wireless link in Adhoc networks. Routing plays an important role of moving the data from source to destination i.e. takes place in the network layer of Open System Interconnection (OSI) reference model. Routing is divided into two techniques i.e. Static routing and Dynamic Routing [3], [5]. In static routing, the routing is done manually, whereas in the dynamic routing, the routing it is an interior and exterior routing protocols. Wireless Local area network provides high speed data transmission which can be accessed from any location. Applications like video conferencing, voice chatting, file transferring can be done in Wireless Local Area network with high transmission speed [1], [7]. In this paper, a Wireless Local Area Network (WLAN) is designed and various routing protocols like Routing Information Protocol (RIP), Enhanced Interior Gateway Routing Protocol (EIGRP), Open Shortest Path First (OSPF), and Interior Gateway Routing Protocol (IGRP) are used and compared for testing the performance [9].

\section{Literature Survey}

Performance analysis of Wireless Local Area Network (WLAN) was performed by using OPNET simulator earlier through delivery traffic. Several authors have given suggestions how to improve the performance e.g. increasing the buffer size. Using high priority traffic, throughput of Wireless Local Area Network (WLAN) is evaluated [5]. In this section, we summarize the basic procedure assumed in multicast protocols and then the Adhoc routing protocols are proposed in the literature survey. Multicast protocols which include the tree-based protocol and mesh-based protocol in which the tree based protocols construct a tree structure for forwarding the packets more efficiently among the neighbors. The work in [2], [3], attempts to improve the transmission i.e. data over a large network without any link breaks. As the focus of our approach, a comparison with routing protocols for Adhoc networks is done by using OPNET simulator tool, so that the performance metrics like packet delivery, delay and throughput are measured for a large network [6]. The HRPM [5] and SPBM [7] are more related work in which the design ideas like decomposition of large groups and distributed geographic hashing to construct a table for better data transfer. Different from general multicasting, the destination are groups of receivers to which all the destinations receive the packet with constant rate depending upon the various routing protocols [9].

\section{Routing Protocols}

In computer networks, the routing protocol specifies how routers communicate to select the routes for information or data transfer for that, the routing algorithm is more important [7]. First, the routing protocol informs or shares the information with their associative neighbors and then throughout the network, in which topology is determined [5] - [10]. Different types of routing protocols are as follows,

$O S P F \& I S-I S->$ Interior gateway routing using link state routing protocol 
$R I P \& E I G R P$

$B G P$
-> Interior gateway routing using Distance vector routing protocol

-> Exterior gateway routing using path vector routing protocol

\subsection{Routing Information Protocol (RIP)}

RIP stands for Routing Information Protocol in which distance vector routing protocol is used for data/packet transmission. In Routing Information protocol (RIP), the maximum number of Hop is 15 , because it prevents routing loops from source to destination. Mechanism like split horizon, route poisoning and holdown are used to prevent from incorrect or wrong routing information. Sally Floyd and Van Jacobson [1994] suggest that, without slight randomization of the timer, the timers are synchronized overtime [6]. Compared to other routing protocol, RIP (Routing Information Protocol) is poor and limit size i.e. small network. The main advantage of using RIP is it uses the UDP (User Datagram Protocol) and reserved port is 520 [10].

\subsection{Enhanced Interior Gateway Protocol (EIGRP)}

EIGRP stands for Enhanced Interior Gateway Protocol which allows router to share information to the neighboring routers which are within the same area. Instead of sending the entire information to the neighboring router, the information which is needed are shared which reduces the workload and amount of data needs to be transmitted. EIGRP (Enhanced Interior Gateway Protocol) designed by CISCO system which can be used only in CISCO routers, but in 2013 it became open source, so it can be used in other routers [5] -[7]. Neighbor table and Topology table are maintained by the EIGRP (Enhanced Interior Gateway Protocol) [10].

\subsection{Open Shortest Path First (OSPF)}

OSPF stands for Open Shortest Path First which uses link-state routing algorithm. Using the link state information which is available in routers, it constructs the topology in which the topology determines the routing table for routing decisions [7]. It supports both variable-length subnet masking and classless inter-domain routing addressing models. Since it uses Dijkstra's algorithm, it computes the shortest path tree for each route. The main advantages of the OSPF (Open Shortest Path first) is that it handles the error detection by itself and it uses multicast addressing for routing in a broadcast domain [8].

\subsection{Intermediate-System to Intermediate - System (IS- IS)}

IS-IS stands for Intermediate-system to Intermediate - system which uses link-state routing algorithm for high speed data transmission. IS-IS (Intermediate-system to Intermediate system) uses Dijkstra's algorithm in which independent database built by each IS-IS router for computing the best path for transmission in a network. It is standardized by ISO, but later IETF (Internet Engineering Task Force) standardized as the Internet Standard in RFC 1142 [3], [6], [10].

\subsection{Interior Gateway Routing Protocol (IGRP)}

IGRP stands for Interior Gateway Routing protocol which uses distance vector protocol (interior) to exchange data within a system [4]. It supports multiple metrics for each node which includes delay, load and bandwidth, in order to compare the 2 routes which are combined into single metrics. The port number for IGRP is 9 which are used for communication and by default every 90 seconds it updates the routing information [5].

\subsection{Comparison of Routing Protocols}

\begin{tabular}{|c|c|c|c|c|c|c|c|}
\hline & RIPv1 & RIPv2 & IGRP & EIGRP & OSPF & IS-IS & BGP \\
\hline Interior/Exterior? & Interior & Interior & Interior & Interior & Interior & Interior & Exterior \\
\hline Type & Distance Vector & Distance Vector & Distance Vector & Hybrid & Link-state & Link-state & Path Vector \\
\hline Default Metric & Hopcount & Hopcount & Bandwidth/Delay & Bandwidth/Delay & Cost & Cost & Multiple Attributes \\
\hline Administrative Distance & 120 & 120 & 100 & $\begin{array}{l}90 \text { (internal) } \\
170 \text { (external) }\end{array}$ & 110 & 115 & $\begin{array}{c}20 \text { (external) } \\
200 \text { (internal) } \\
\end{array}$ \\
\hline Hopcount Limit & 15 & 15 & 255 (100 default) & 224 (100 default) & None & None & $\begin{array}{l}\text { EBGP Neighbors: } 1 \text { (default) } \\
\text { IBGP Neighbors: None }\end{array}$ \\
\hline Convergence & Slow & Slow & Slow & Very Fast & Fast & Fast & Average \\
\hline Update timers & 30 seconds & 30 seconds & 90 seconds & Only when change occurs & $\begin{array}{c}\text { Only when changes occur, } \\
\text { (LSA table is refreshed every } 30 \\
\text { minutes, however) }\end{array}$ & Only when changes occur & Only when changes occur \\
\hline Updates & Full table & Full table & Full table & Only Changes & Only Changes & Only changes & Only changes \\
\hline Classless & No & Yes & No & Yes & Yes & Yes & Yes \\
\hline Supports VLSM & No & Yes & No & Yes & Yes & Yes & Yes \\
\hline Algorithm & Bellman-Ford & Bellman-Ford & Bellman-Ford & DUAL & Dijkstra & Dijkstra & Best Path Algorithm \\
\hline Update Address & Broadcast & 224.0 .0 .9 & 224.0 .0 .10 & 224.0 .0 .10 & $\begin{array}{l}224.0 .0 .5 \text { (All SPF Routers) } \\
224.0 .0 .6 \text { (DR's and BDR's) }\end{array}$ & & Unicast \\
\hline Protocol and Port & UDP port 520 & & IPProtocol 9 & IP Protocol 88 & IP Protocol 89 & & TCP port 179 \\
\hline
\end{tabular}

"Table." 1 Comparison of routing protocols 


\section{Performance Metrics}

* End to End Delay -> Sum of the node delay at each node + link delay at each link on the path

$$
\frac{\sum_{1}^{n}\left(C B R_{-} \text {sent_time }-C B R_{-} \text {receive_time }\right)}{\sum_{1}^{n} C B R_{-} \text {received }},
$$

* Throughput -> Total size of packets received at destination nodes which measured in Kbps (kilo bits per second)

* Packet delivery _> Ratio of data packets delivered to the destination generated by CBR.

$$
P D R[\%]=\frac{\sum_{i}^{n} C B R_{-} \text {received }}{\sum_{1}^{m} C B R_{-} \text {sent }} \times 100,
$$

Routing -> Ratio of routing protocol to the total number of packets generated by the source.

$$
N R L=\frac{\sum_{1}^{k} \text { Routing_packets }}{\sum_{1}^{n} C B R \text { _received }},
$$

\section{Experimental Setup And Result Analysis}

In this paper, the proposed network has been simulated by using OPNET simulator tool and the performances of different routing protocols were analyzed. Components for the network design, WLAN parameters and profile configuration parameters are used in our experiment are shown in table 1, table 2 and table 3 respectively. The performance like load, delay, throughput for various protocols were taken and analyzed.

\begin{tabular}{|c|c|}
\hline COMPONENT & MODEL \\
\hline Application Configuration & Application Config \\
\hline Profile Configuration & Profile Config \\
\hline Ethernet Server & 10BaseT connector \\
\hline Connection & Wlan_ethernet_router \\
\hline Access Point & Wlan_wkstn_adv \\
\hline Nodes & RIP, EIGRP, IGRP, OSPF \\
\hline Protocol & \\
\hline
\end{tabular}

"Table." 2 Components for the network design

\begin{tabular}{|c|c|}
\hline ATTRIBUTE & VALUE \\
\hline BSS Identifier & Auto Assigned \\
\hline Access Point Functionality & Enabled \\
\hline Buffer size (bits) & $\mathbf{2 5 6 0 0 0}$ \\
\hline Data rate (bps) & $\mathbf{1 M b p s , 1 1 M b p s}$ \\
\hline
\end{tabular}

“Table.” 3 WLAN parameters

\begin{tabular}{|c|c|}
\hline ATTRIBUTE & VALUE \\
\hline Profile Configuration & Profile Config \\
\hline Profile name & Web application, Wireless application \\
\hline Operation mode & Serial (ordered) \\
\hline Start time & Uniform(100,110) \\
\hline Duration & End of Simulation \\
\hline Repeatability & Once a start time \\
\hline
\end{tabular}

"Table." 4 Profile Configuration parameters 


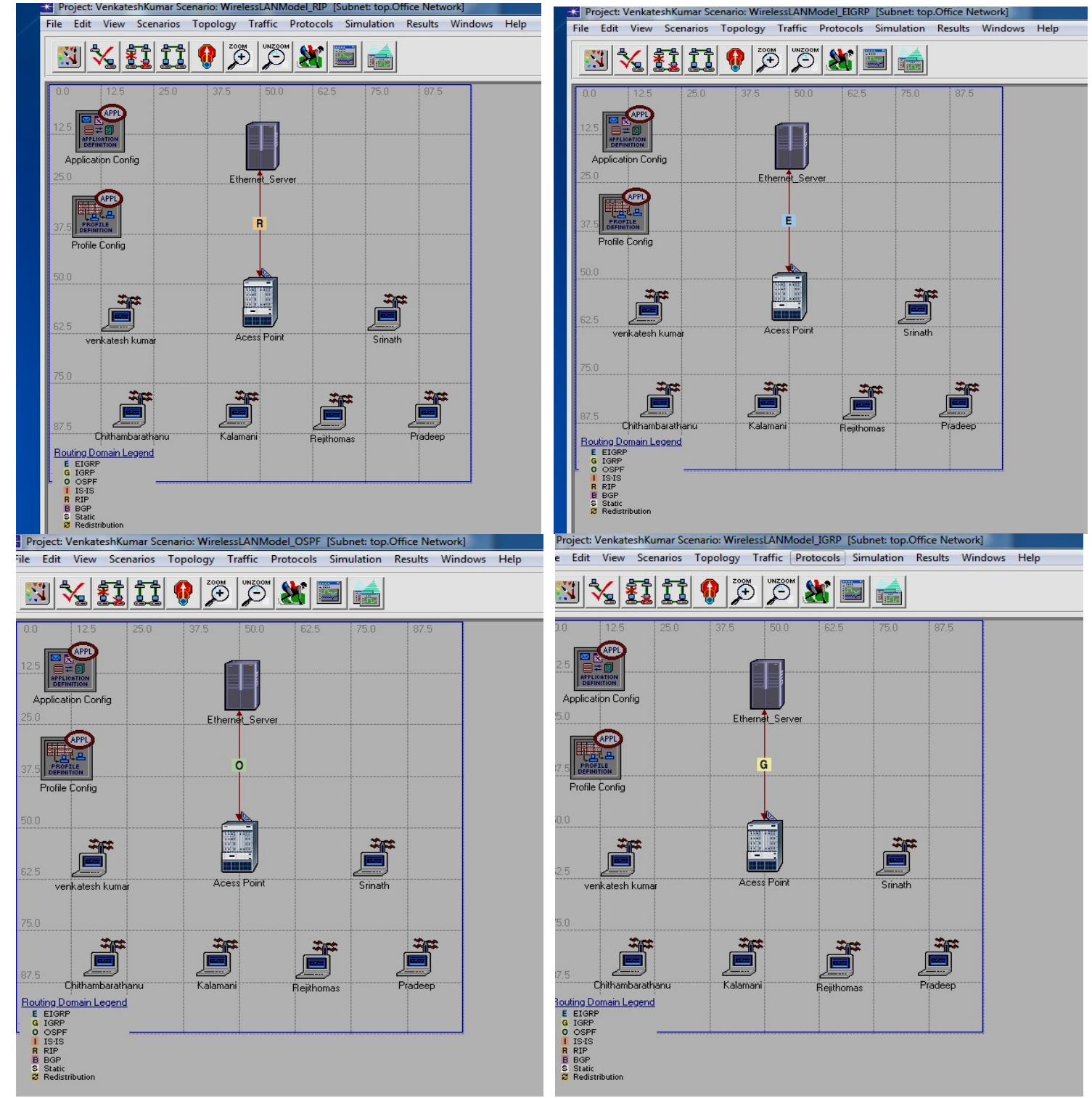

"Fig." 1, 2, 3 and 4 represents final design network of RIP (Top Left), EIGRP (Top Right), OSPF (Bottom left) and IGRP (Bottom Right)

\subsection{Result analysis}

In this section, the procedure for collection of statistics and the execution performance are described which collected from individual nodes i.e. node statistics and global statistics. The following results like simulation sequence diagram, delay, throughput, packet delivery, Ethernet delay, traffic send /receive were analyzed.

\subsubsection{Sequence Simulation}

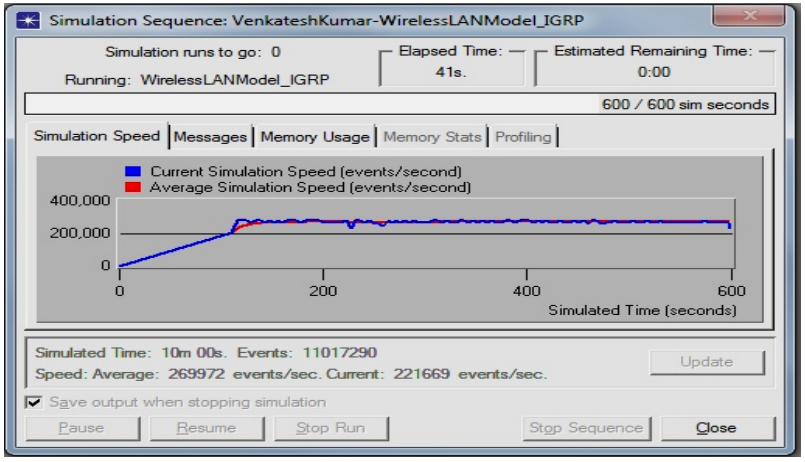

"Fig." 5 sequence simulation of IGRP 


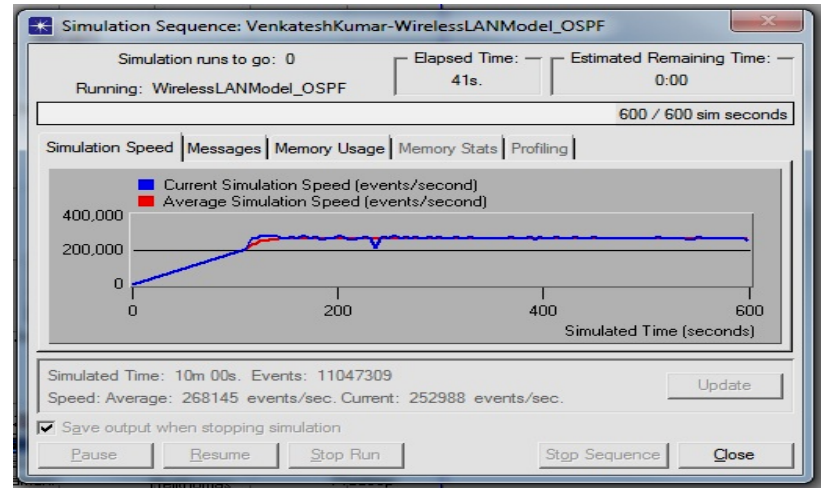

"Fig." 6 sequence simulation of OSPF

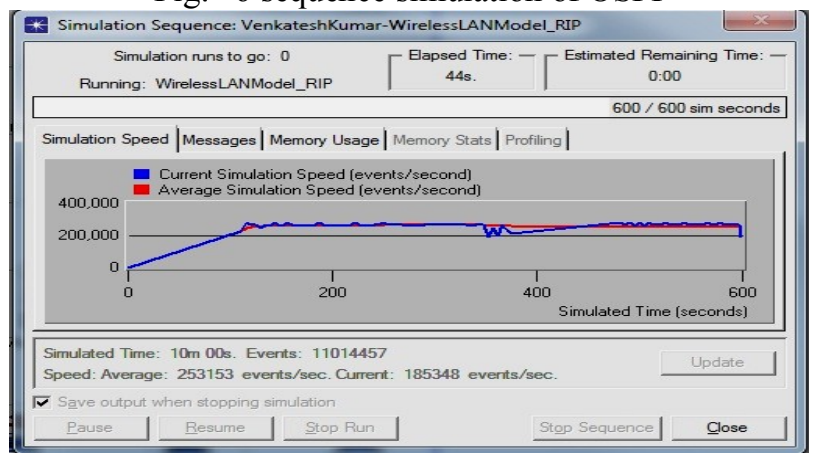

"Fig." 7 sequence simulation of RIP

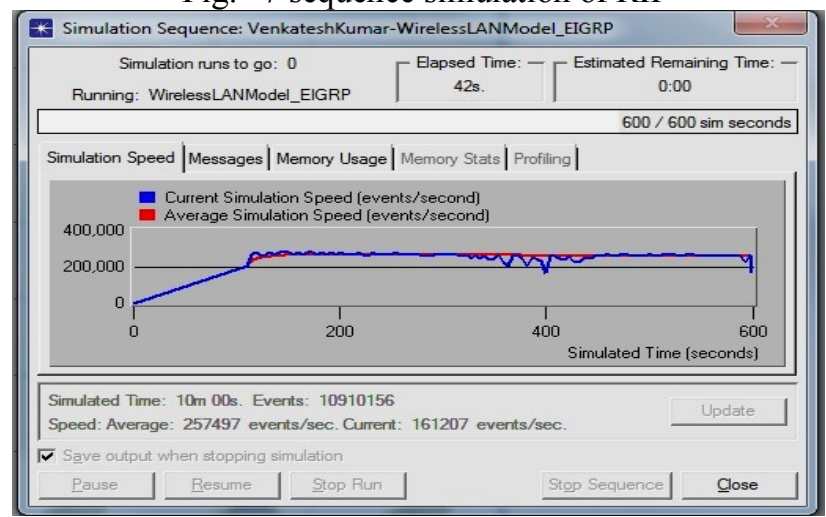

"Fig." 8 sequence simulation of EIGRP

\subsubsection{Ethernet Delay}

It represents the end-to-end delay of all packets received by all the nodes. The average time Ethernet delay of the entire network for different values percentages is observed. We can analyze that the average time delay increases as the back utilization increases [4].
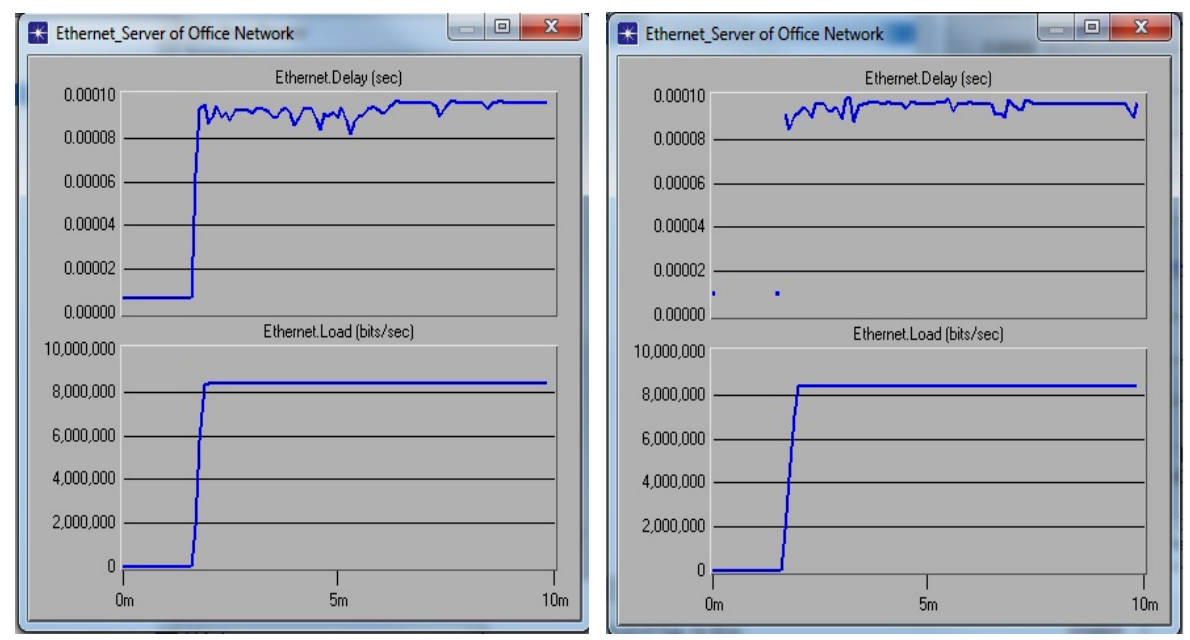

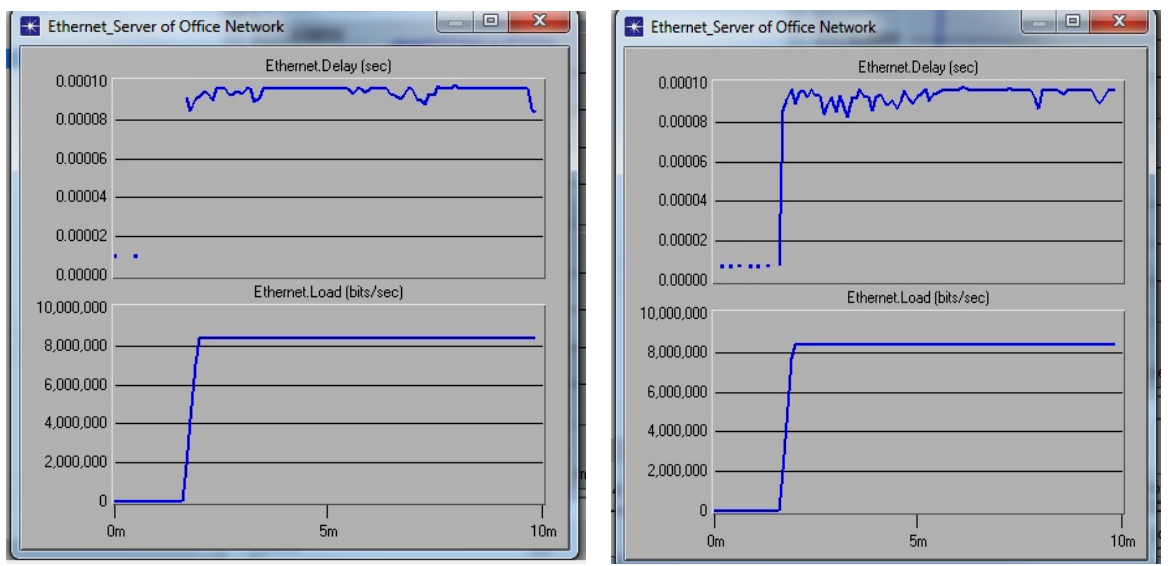

"Fig." 9, 10, 11, 12 Ethernet delay of EIGRP (Top Left), IGRP (Top Right), OSPF (Bottom Left), RIP (Bottom Right)

\subsubsection{WLAN Delay (Access Point)}

Delay is gathered for 10 minutes of simulation time for different routing protocols. Delay represents the end-to-end delay of packets which is received by the WLAN MAC's of all the nodes which is forwarded to the higher layer [1] - [5]. The access point is enabled, so that the delay includes the medium access delay at the source MAC. At $1 \mathrm{Mbps}$ data rate, the end-to-end delay is increasing when compared to the $11 \mathrm{Mbps}$ data rate. More data rate reduces the delay which is shown in the figure [13, 14, 15, and 16].

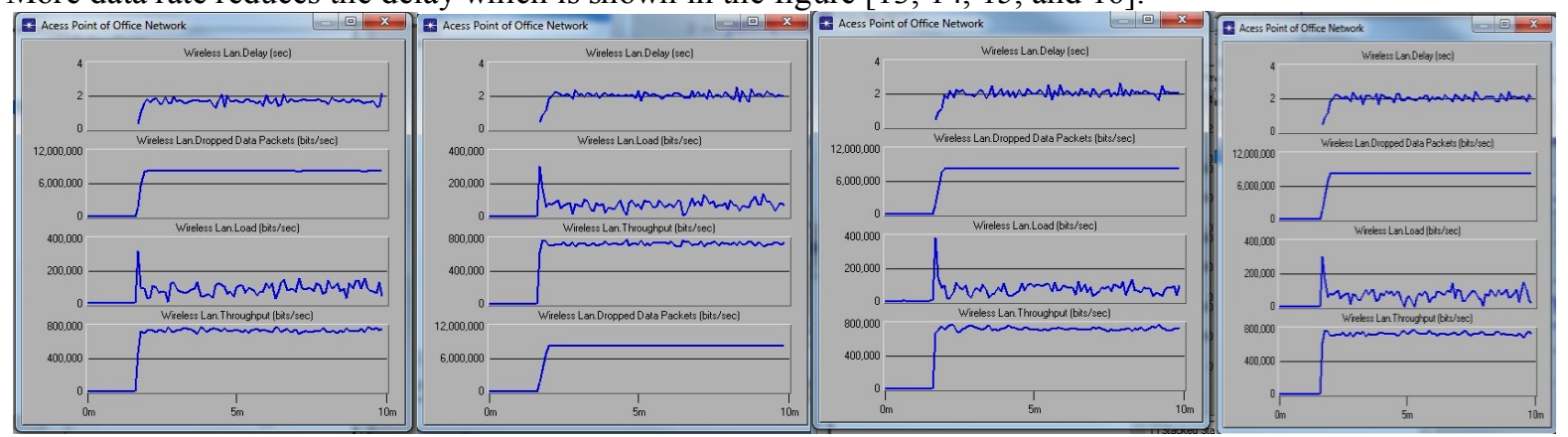

"Fig." 13, 14, 15, 16 Delay of EIGRP, IGRP, OSPF, RIP (Left to Right)

\subsubsection{Load and Throughput}

The generation rate of data is $1 \mathrm{Mbps}$ according to the proposed system and the relationship between the load and throughput is discussed in this section. Load is defined as the total load submitted to the WLAN nodes in (bits/sec) where as, the throughput is total number of bits forwarded from wireless LAN to all WLAN nodes in the network. From the figure. [17-24] shows the load is greater than the throughput for different routing protocols and the load is fixed, so that the throughput is improved at different data rates.

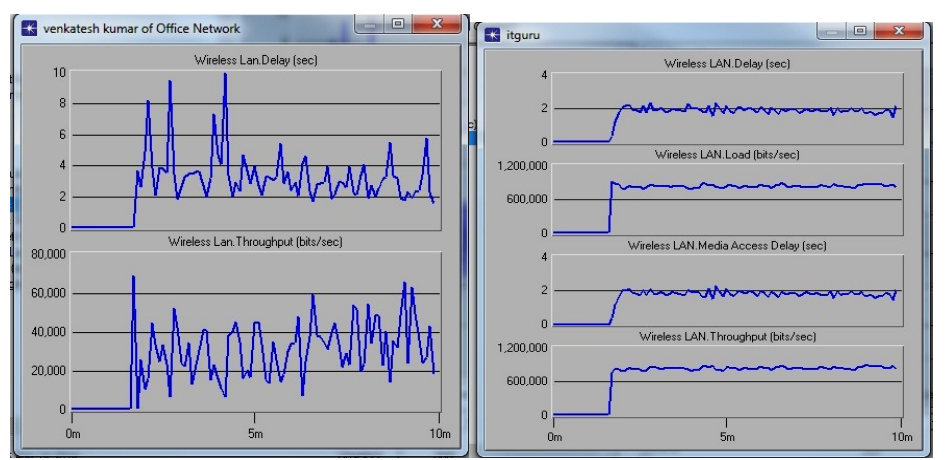




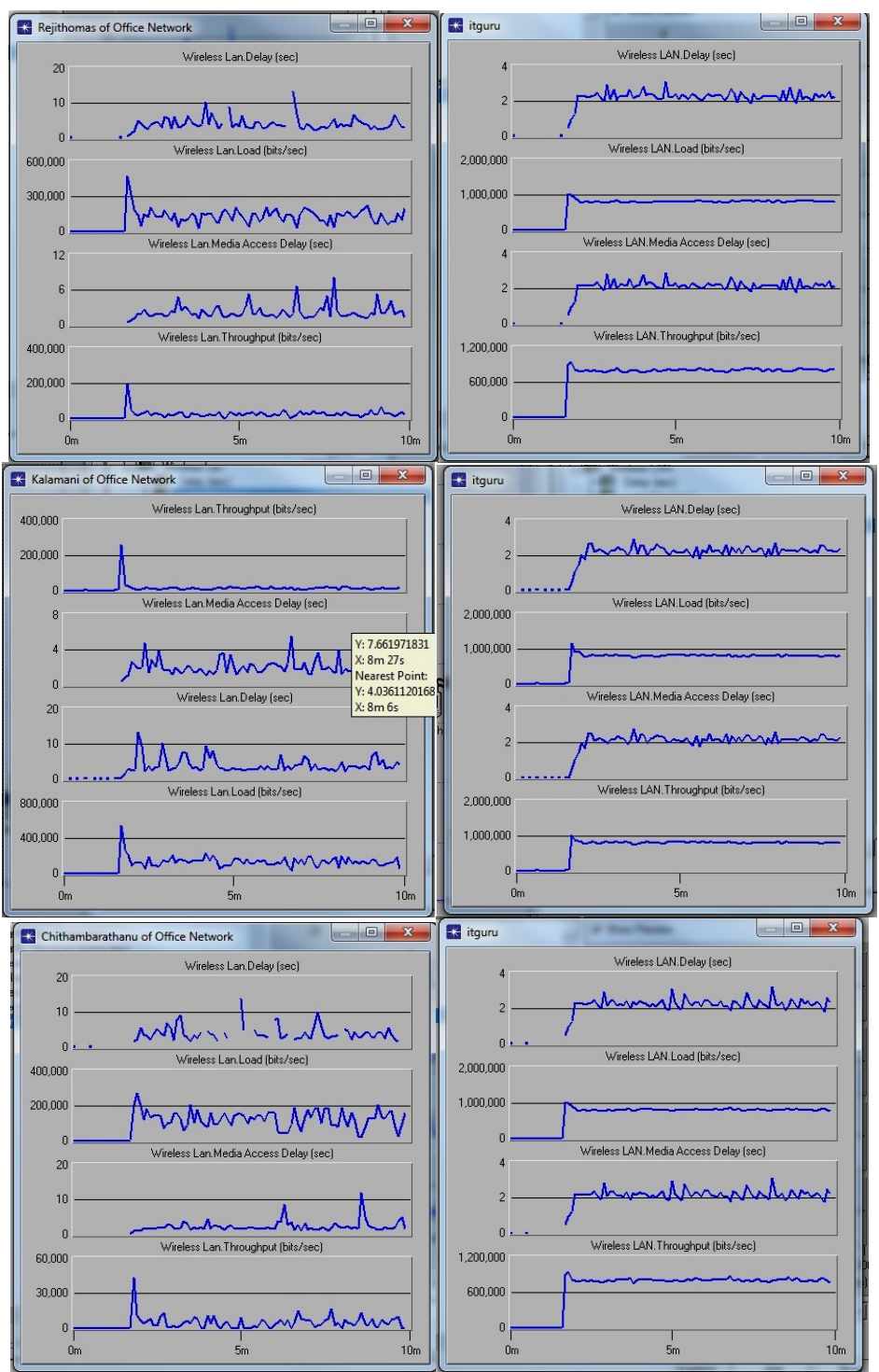

"Fig 17, 18, 19, 20, 21, 22, 23, 24 Load \& Throughput

\section{Feasibility Study}

In the feasibility study, three considerations like economical feasibility, technical feasibility and social feasibility are analyzed.
Economic feasibility
$\rightarrow$ Cost
Technical feasibility
-> Requirements \& Resources
Social feasibility
$\rightarrow$ Protection \& Security

\section{Conclusion}

The main objective of this paper is about the WLAN (Wireless Local Area Network) and their technologies, routing protocols and operation modes. In this paper, the performance of WLAN is evaluated by using OPNET simulator and performance metrics like load, delay, throughput, packet delivery were obtained for different routing protocols like EIGRP, RIP, IGRP and OSPF. From the result, we analyzed that the delay is improved by increasing the transmission rate. EIGRP and OSPF is more efficient than other routing protocols in terms of throughput and load. A comparison between different protocols were analyzed and we can suggest that markets like large enterprises, educational institutes, industrial sites can implement EIGRP and OSPF routing protocol for better performance and key catalyst like 802.11a, 802.11g can accelerate the WLAN(Wireless Local Area Network) with the speed upto 54Mbps [2] - [7]. 


\section{Journal Papers:}

\section{References}

[1] S. Shah, et al., "Performance Evaluation of Ad Hoc Routing Protocols Using NS2 Simulation," Proceedings of the National Conference on Mobile and Pervasive Computing (CoMPC-2008), Chennai, India, August 2008.

[2] K. Gorantala, "Routing Protocols in Mobile Ad Hoc Networks," Master Thesis, Department of Computing Science, Umeøa University, Sweden, June 2006.

[3] Z. Bojković, M. Stojanović, and B. Milovanović, “Current Developments towards the 4G Wireless System," Proceedings of International Conference TELSIKS, Niš, Serbia, September 2005, pp. 229-232.

[4] S. Barakovićand J. Baraković, “Comparative Performance Evaluation of Mobile Ad Hoc Routing Protocols, ”Proceedings of the $33^{\text {rd }}$ International Convention on Information and Communication Technology, Electronics and Microelectronics (MIPRO 2010), Opatija, Croatia, May 2010.

[5] Nurul I. Sarkar \& Wilford G. Lol “A Study of MANET Routing Protocols: Joint Node Density, Packet Length and Mobility” 978-14244-7755-5/10/\$26.00 (C2010 IEEE Page no. 515-520

[6] Vasudha Arora \& C. Rama Krishna "Performance Evaluation of Routing Protocols for MANETs under Different Traffic Conditions" 2010 2nd International Conference on Computer Engineering and Technology [Volume 6] 978-1-4244-63497/10/\$26.00 c 2010 IEEE

[7] Patel, B.; Srivastava, S.; "Performance analysis of zone routing protocols in Mobile Ad Hoc Networks," Communications (NCC), 2010 National Conference on, vol.,pp.1-5, 29-31 Jan. 2010.

[8] J. Wang, F. Xu, F. Sun. “Benchmarkinng of Routing Protocols for Layered Satellite Networks”. In Proceedings of Multiconference on Computational Engineering in Systems Applications, pp. 1087-1094, vol. 2, Oct 2006.

[9] Lachhman,S., Asad, Y., Malkani "Performance analysis of WLAN standards for video conferencing applications", International Journal of Wireless \& Mobile Networks (IJWMN) Vol. 3, No. 6, December 2011

[10] Rajan, R., Shipra, S. "WLAN Performance Improvisation by Fine Tuning IEEE 802.11 Parameters", International Journal of Computer Applications, April 2012. 